Submitted: 30/01/2017

Accepted: 15/06/2017

Published: 26/06/2017

\title{
Expression of various sarcomeric tropomyosin isoforms in equine striated muscles
}

\author{
Syamalima Dube ${ }^{1}$, Henry Chionuma ${ }^{1}$, Amr Matoq ${ }^{2}$, Ruham Alshiekh-Nasany ${ }^{1}$, Lynn Abbott $^{1}$, Bernard J. Poiesz ${ }^{1}$ \\ and Dipak K. Dube ${ }^{1, *}$ \\ ${ }^{1}$ Department of Medicine, SUNY Upstate Medical University, 750 East Adams Street, Syracuse, New York 13210, \\ USA \\ ${ }^{2}$ University of Florida, College of Medicine-Jacksonville, Suite 1130, 841 Prudential Drive, Jacksonville, FL 32207, \\ USA
}

\begin{abstract}
In order to better understand the training and athletic activity of horses, we must have complete understanding of the isoform diversity of various myofibrillar protein genes like tropomyosin. Tropomyosin (TPM), a coiled-coil dimeric protein, is a component of thin filament in striated muscles. In mammals, four TPM genes (TPM1, TPM2, TPM3, and TPM4) generate a multitude of TPM isoforms via alternate splicing and/or using different promoters. Unfortunately, our knowledge of TPM isoform diversity in the horse is very limited. Hence, we undertook a comprehensive exploratory study of various TPM isoforms from horse heart and skeletal muscle. We have cloned and sequenced two sarcomeric isoforms of the TPM1 gene called TPM1 $\alpha$ and TPM1 the TPM3 gene, TPM $2 \alpha$ and TPM3 $\alpha$ respectively. By qRT-PCR using both relative expression and copy number, we have shown that TPM1 $\alpha$ expression compared to TPM1 $\kappa$ is very high in heart. On the other hand, the expression of TPM1 $\alpha$ is higher in skeletal muscle compared to heart. Further, the expression of TPM $2 \alpha$ and TPM $3 \alpha$ are higher in skeletal muscle compared to heart. Using western blot analyses with $\mathrm{CH} 1$ monoclonal antibody we have shown the high expression levels of sarcomeric TPM proteins in cardiac and skeletal muscle. Due to the paucity of isoform specific antibodies we cannot specifically detect the expression of TPM1 $\kappa$ in horse striated muscle. To the best of our knowledge this is the very first report on the characterization of sarcmeric TPMs in horse striated muscle.
\end{abstract}

Keywords: Absolute copy number, Horse, qRT-PCR, Relative expression, TPM.

\section{Introduction}

Tropomyosins, a family of actin-biding proteins, are present in all eukaryotes from yeast to humans. They play a critical role(s) in regulating the function of actin filaments in both muscle and nonmuscle cells (LeesMiller and Helfman, 1991; Pittenger et al., 1994; Pieples and Wieczorek, 2000; Perry, 2001; Denz et al., 2004; Gunning et al., 2008).

Sarcomeric tropomyosin is a component of the thin filament whereas muscle myosin isoforms comprise the thick filament in myofibrils. The dynamic interaction between the thick and thin filaments results in muscle contraction, which is triggered by nerve impulses that in turn stimulate the release of $\mathrm{Ca}^{2+}$ in the cytosol. Tropomyosin attached troponin(s) then binds to $\mathrm{Ca}^{+2}$ and undergoes a massive conformational change that aids the myosin associated ATPase activity to hydrolyze ATP and release energy. The energy thus released initiates muscle contraction. Muscle contraction is a function that various animals, including humans, exploit to move, stretch, and even keep themselves warm. Hence, various myofibrillar proteins including tropomyosin play critical role(s) in muscle contraction that is essential for walking, running, and exercising.

The horse is a unique and incredible athlete with remarkable capabilities. In fact, athletic performance is a vital criteria used for the selection of superior horses. It is well established that athletic performance of animals including humans can be improved by proper and rigorous physical exercise/training, which again depends on the expression of various genes related to energy metabolism as well as muscle contraction (McGivney et al., 2009; Eivers et al., 2010, 2012). However, very little is known about exercise-related gene expression patterns in equine muscles for example heart, and skeletal muscles.

Some myofibrillar protein genes like, TNNC2 (Tn-C type 2) ACTN1 (alpha actin, skeletal muscle) and TPM1 (tropmyosin 1) were found to be up regulated in equine skeletal muscle after exercise (Eivers et al., 2012). TPM1 and TPM 2 along with the transcripts of some other myofibrillar protein genes are among the most highly abundant transcripts in equine skeletal 
muscle (McGivney et al., 2010). Yet, to the best our knowledge, none of the sarcomeric TPM isoforms from equine heart and skeletal muscles have been cloned or sequenced. Nor have extensive studies been done to evaluate the full repertoire of TPM sarcomeric isoforms expressed in equine cardiac and skeletal muscles.

In vertebrates, tropomyosin is encoded by four tropomyosin genes TPM1, TPM2, TPM3, and TPM4 (Lees-Miller and Helfman, 1991; Pittenger et al., 1994; Pieples and Wieczorek, 2000; Perry, 2001; Denz et al., 2004; Gunning et al., 2008) except for zebrafish where six tropomyosin genes are present (Booth et al., 1998; McGivney et al., 2010). In mammalian systems the TPM sarcomeric isoforms deemed to be important in heart and skeletal muscle includeTPM1 $\alpha$, ТРM1к, TPM $2 \alpha$, and TPM $3 \alpha$ (Lees-Miller and Helfman, 1991; Perry, 2001; Gunning et al., 2008; Dube et al., 2016). Hence, in this study, we have cloned and sequenced the above sarcomeric tropomyosin cDNAs from equine striated muscles. Recently, we have described sarcomeric TPM $4 \alpha$ expression in human hearts and, to a much more limited degree, human skeletal muscle (Dube et al., 2016).

However, in several mammals, for example rodents, the TPM4 gene is truncated. As a result, the TPM4 gene does not code a sarcomeric isoform in rodents. Also, the predicted isoforms of the horse TPM4 gene (GenBank mRNA accession \# XM_014734753.1) did not include the sarcomeric isoform because of a stop codon in exon 2. Further, no definitive role on muscle contraction in mammals has yet been assigned for TPM $4 \alpha$, the sarcomeric isoform of the TPM4 gene. Hence, in this study, we have not included TPM $4 \alpha$.

\section{Materials and Methods}

Conventional and nested RT-PCR for amplification of TPM1 $\alpha, T P M 1 \kappa, T P M 2 \alpha$ and TPM3 $\alpha$

Total RNAs from whole horse (Equus ferus caballus) heart and skeletal muscle were procured from Zyagen (San Diego, CA) and BioChain (Newark, CA). 1st strand cDNA was made using oligo (dT)12-15 using SuperScript II (Life Technologies, Grand Island, NY) following manufacturer's specifications as described earlier (Thurston et al., 2009; Nan et al., 2015). First, PCR amplification for TPM $1 \alpha$ and TPM1 $\kappa$ were carried out with the $\mathrm{P} 1$ and $\mathrm{P} 2$ primer-pair (Table 1 and Fig. 1).

For nested PCR, the first amplified DNAs from heart and skeletal muscle were diluted 200 fold with distilled water and $2 \mu \mathrm{l}$ from each sample was amplified with the P5 and P2 primer-pair for TPM1 $\kappa$ amplification and P3 and P2 primer-pair for TPM1 $\alpha$ amplification. P8 was used as probe for both isoforms. The primer combinations P5 and P6; P1 and P7 were used in the direct sequencing of TPM1 $\kappa$. Nucleotide sequences of various primer-pairs used for amplification of different isoforms are provided in Table 1.

Following amplification the DNA product was run on a $1.5 \%$ agarose gel. DNA bands of appropriate size(s) were extracted using the MinElute Gel Extraction kit provided by QIAGEN. One portion of isolated DNA was used for determination of nucleotide sequences and a portion of the isolated DNA was ligated into a T/A cloning vector (Invitrogen) using the manufacturer's specifications as previously described (Thurston et al., 2009; Nan et al., 2015).

This resulting ligation mixture was then used for the transformation of competent One Shot E. coli cells (provided by Invitrogen) as per the manufacturer's protocol. For identification of the particular isoform we performed colony hybridization with $\left[{ }^{32} \mathrm{P}\right]$-labeled Exon $2 b$ specific probe for TPM1 $\alpha$ or exon $2 a$ specific probe for TPM1 $\kappa$, as listed in Table 1. The hybridization positive colonies were picked up; grown overnight and plasmid DNA were isolated using the QIAprep Spin Miniprep kit (QIAGEN). The isolated plasmid DNA as well as the gel extracted DNA (as stated above) were sequenced at the Cornell University Core DNA sequencing facility. For amplification of TPM $2 \alpha$ and TPM $3 \alpha$ we employed TPM $2 \alpha$ and TPM $3 \alpha$ specific primer-pairs designed using the predicted sequences available in GenBank. The amplified DNAs were cloned into T/A cloning vector (Invitrogen) and subsequently sequenced as stated above.

\section{Real-time quantitative RT-PCR.}

qRT-PCR analyses of cDNA templates were performed using the LightCycler 480 Real-Time PCR System as described before (Dube et al., 2014; Nan et al., 2015). Reactions were carried out in a 384-well plate using the LightCycler 480 SYBR Green I Master kit (Roche). Briefly, each well contained a total volume of $10 \mu \mathrm{l}$ reaction solution, of which $2 \mu \mathrm{l}$ was cDNA template and $8 \mu \mathrm{l}$ was SYBR green mix $(5 \mu 11 \times$ SYBR green Master mix, $2.8 \mu \mathrm{l}$ of PCR-grade water, and $0.2 \mu \mathrm{l}$ of $10 \mu \mathrm{M}$ primer pair). Primers for real-time PCR for various TPM isoforms are listed in Table 1. In case of TPM1 $\alpha$ and TPM1 $\kappa$, cDNA was made with an oligonucleotide specific for exon $9 \mathrm{a} / \mathrm{b}$ that allowed us to make an isoform specific cDNA corresponding to the mRNA containing exon $9 \mathrm{a} / \mathrm{b}$ as in various sarcomeric isoforms. Amplification in the absence of a cDNA template was also evaluated to insure a lack of signal due to primer dimerization and extension or carryover. Data were analyzed using both relative and absolute quantification methods. Relative quantification of qRTPCR data was performed using the delta CT (sample CT minus 18S rRNA CT) and delta delta CT (sample delta CT minus comparator delta CT) methods (Pfaffl, 2001; Livak and Schmittget, 2001). 


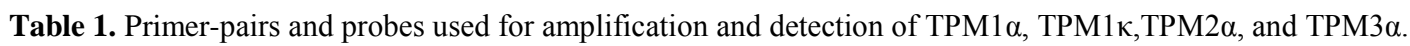

\begin{tabular}{|c|c|c|c|c|}
\hline \multirow{2}{*}{ 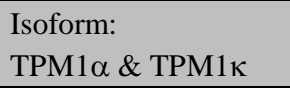 } & \multirow{2}{*}{$\begin{array}{l}\text { As indicated in } \\
\text { Fig. } 1\end{array}$} & \multicolumn{2}{|c|}{ Type of amplification } & \multirow[b]{2}{*}{ Nucleotide sequence } \\
\hline & & Con RT-PCR & qRT-PCR & \\
\hline MTPM1-1A(+) & $\mathrm{P} 1$ & $\mathrm{X}$ & - & $5^{\prime}$-ATGGACGCCATCAAGAAGAA-3' \\
\hline MTPM1-2A(+) & P5 & $\mathrm{X}$ & & $5^{\prime}-$ CGGAGGACGAGCGGGACCGGG-3' \\
\hline MTPM1-2A probe & P4 & & $\mathrm{X}$ & $5^{\prime}$-GAGCTGCACAAGGCGGAGGACAG-3' \\
\hline MTPM1-2B (+) & P3 & & $\mathrm{X}$ & $5^{\prime}$-ATGAACTGGACAAATACTCTGAG-3' \\
\hline MTPM1-2A(-) & P7 & & & $5^{\prime}$-TGTCCTCCGCCTTGTGCAG-3' \\
\hline MTPM1-3-4(-) & P6 & & $\mathrm{X}$ & $5^{\prime}$-TCAATGACTTTCATGCCTCT-3' \\
\hline MTPM1-9A(-) & $\mathrm{P} 2$ & $\mathrm{X}$ & & $5^{\prime}$-CGCTCTCAACGATATGACTT-3' \\
\hline \multicolumn{5}{|l|}{ TPM $2 \alpha$} \\
\hline MTPM2-1A(+) & & $\mathrm{X}$ & & $5^{\prime}-$ ATGGACGCCATCAAGAAGAAG-3' \\
\hline MTPM2-9A(-) & & $\mathrm{X}$ & & $5^{\prime}$-TCAGAGGGAGGTGATGTCATTGA-3' \\
\hline TPM2 (+) & & & $\mathrm{X}$ & $5^{\prime}-$ CTCAAGGAGGCAGAGACCCG-3' \\
\hline TPM2 (-) & & & $\mathrm{X}$ & $5^{\prime}$-GGCCACACTGGTGGGGGCTC-3' \\
\hline MTPM2 Exon 3 probe & & & & $5^{\prime}$-ATTCAGCTGGTTGAGGAGGAGCTGG-3' \\
\hline \multicolumn{5}{|l|}{ TPM $3 \alpha$} \\
\hline MTPM3-1A(+) & & $\mathrm{X}$ & & $5^{\prime}$-CGCCTGGCCACTGCCCTGCAA-3' \\
\hline MTPM3-9A(-) & & $\mathrm{X}$ & & $5^{\prime}$-GAGTCTGGTCCAGCATCCTT-3' \\
\hline TPM3 (+) & & & $\mathrm{X}$ & 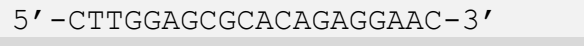 \\
\hline TPM3 (-) & & & $\mathrm{X}$ & $5^{\prime}$-GATCCAGAACAGAGCAGAAAC-3' \\
\hline MTPM3 Exon 2 probe & & & & 5'-GAGAAGAAGGCTGCTGAT-3' \\
\hline
\end{tabular}

A comparative value was calculated using the formula $\mathrm{X}^{\text {-delta delta CT }}$, where " $E$ " equals the efficiency of specific primer pairs. This is similar to the $2^{\text {-delta delta } \mathrm{CT}}$ method but corrects for the assumption that the reaction is occurring with $100 \%$ efficiency. Efficiencies $(E)$ were determined using dilution series of isoform-specific plasmid clones with respective isoform-specific primers pairs.

The LightCycler 480 software plotted the CT at each concentration against the logarithm of the dilutions of the clone, generating a linear regression curve that calculated efficiency based on the formula $E$ $=10^{[-1 / \text { slope }]}$ Efficiency of $18 \mathrm{~S}$ rRNA was determined by serial dilution of horse cDNAs generated with specific primers (Dube et al., 2014; Nan et al., 2015).

For determination of absolute copy number, optical density was taken of various horse isoform specific

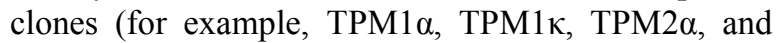
TPM $3 \alpha$ separately using a spectrophotometer. The copy number per volume of clone in solution was determined using the equation number of copies $=(\mathrm{ng}$ of plasmid DNA x $\left.6.02 \times 10^{23}\right) /(\mathrm{bp}$ length of plasmid $\mathrm{x}$ $1 \times 10^{9} \times 650$ ), which was simplified by Andrew Staroscik at the URI Genomics and Sequencing Center. A dilution series of each clone was done for $1 \times 10^{1}-1$ $\mathrm{x} 10^{4}$ copies of template, which was used to create a standard curve after amplification (Booth et al., 1998; Livak and Schmittgen, 2001; Nan et al., 2015). For better accuracy, each sample in the dilution series was run in triplicate.

\section{Western blot}

Horse heart and horse skeletal thigh muscle protein extracts were obtained from ZYAGEN. Protein concentration was verified using the BioRad protein reagent kit. For each sample in a total reaction mixture of $30 \mu \mathrm{l}$ was made, $5 \mu \mathrm{l}$ protein sample, $7.5 \mu \mathrm{l}$ NuPage LDS sample buffer (4x), $15.5 \mu \mathrm{l}$ distilled water and $2 \mu \mathrm{l}$ NuPage sample reducing agent (10x). The samples were run on NuPage $4-12 \%$ Bis Tris gel and then transferred to nitrocellulose membrane according to the manufacturer's instruction as described earlier (Thomas et al., 2010).

The membrane was stained with Ponceau S solution in order to verify the transfer of the protein onto the membrane itself. The Ponceau stained membrane paper was then blocked in $5 \%$ milk protein solution for $\sim 1$ hour at room temperature to prevent non-specific binding. Primary antibody (for example $\mathrm{CH} 1$ or anti GAPDH) with appropriate dilution in $5 \%$ milk was incubated at $4^{\circ} \mathrm{C}$ overnight with constant shaking. The membrane paper was washed three times in TBST solution. Secondary antibody was then applied to the membrane. Secondary antibody used for GAPDH, CH1 was anti-Mouse IgG. After the application of secondary antibody the nitrocellulose membrane was again washed three times with TBST. Electrochemiluminescence (ECL) was applied by using equal amounts of BioRad ClarityTM Western ECL substrates. Imaging was done using BioRad chemi MP Imaging system. In the case that the signal from the 
ECL process was too weak the blot was exposed to $\mathrm{x}$ ray film.

\section{Statistical Analysis}

The means, standard deviations, and comparative analyses of each data set for statistical significance were done using paired Student's $t$-test. Western Blot analyses for protein expression with extracts of various mammalian systems (Yuan, et al., 2006).

\section{Results}

Evaluation of expression of TPM1 and TPM1 $\kappa$ in horse cardiac and skeletal muscles using conventional as well as nested RT-PCR.

Figure 1 shows the exon composition of TPM1 $\alpha$ and ТРМ1к. The only difference between these two TPM1 isoforms is in exon 2. For identification, we first used conventional RT-PCR with a primer-pair that amplifies

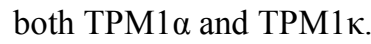

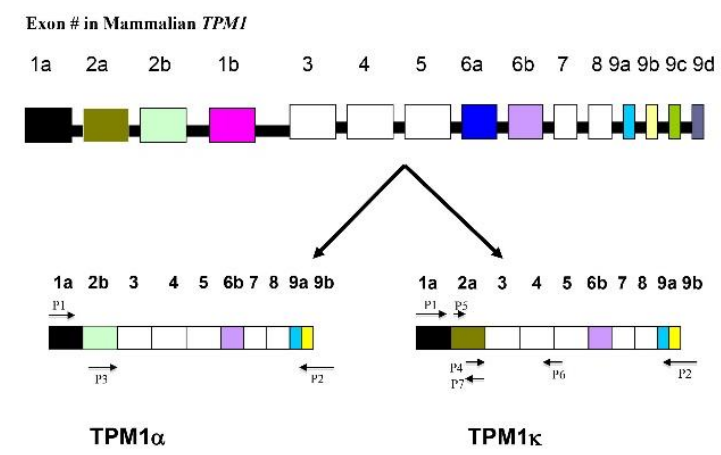

Fig. 1. Alternatively spliced two sarcomeric $T P M 1$ isoforms.

The results presented in Figure 2A (panel a-b) show the amplified DNA in heart (lane 1) and skeletal muscle (lane 2). In the next step, we amplified the previous amplicons with isoform specific primer-pairs as described under methods section. The results in Figure 2B (panel a-b) show that TPM1 $\alpha$ is expressed in both heart (lane 1) and skeletal muscle (lane 2). Similarly, the results in panel Figure 2B (panel c-d) show that ТРМ1 $\kappa$ is also expressed in both cardiac and skeletal muscle. The expression patterns of the two isoforms were further confirmed by determining the nucleotide sequences of the gel extracted DNA.

Further, we cloned the DNA amplified by the generic primer-pair into T/A cloning vector and identified the particular isoform by PCR amplification of the DNA of each colony directly with an isoform specific primerpair. We identified colonies (3 from each isoform), isolated DNA from each colony, and determined nucleotide sequences. The cloned DNAs for each isoform were subsequently used as the template for determining the absolute copy number of the particular transcript expressed in cardiac and skeletal muscle cells by qRT-PCR.
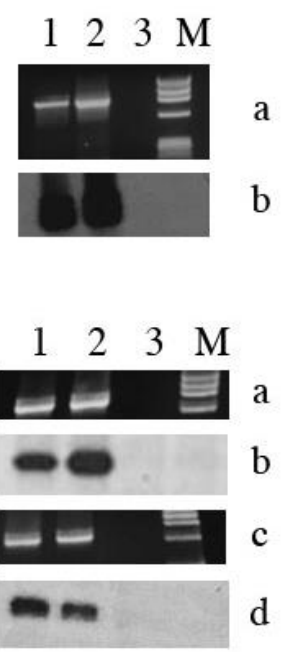

Fig. 2. Agarose gel electrophoresis of amplified TPM1 $\alpha$ and

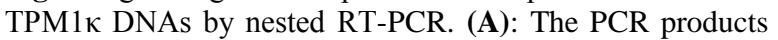
generated by the generic primer-pair MTPM1-

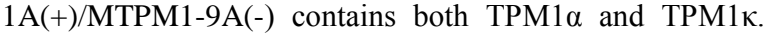
(Panel a): Ethidium bromide staining of the amplified DNA ran onto agarose gel. Panel b. Hybridization with $\left[{ }^{32} \mathrm{P}\right]$ labeled TPM1exon 3 probe. (B): The final amplified DNA (Fig. 2A) was diluted 200 fold and $2 \mu$ of diluted DNA was used for amplification with TPM $1 \alpha$ and TPM1 $\kappa$ specific primer-pairs. (Panel a): The PCR amplified DNA with TPM1 $\alpha$ - specific primer -pair MTPM1-2B (+)/MTPM1-9A(-) were separated by agarose gel electrophoresis and finally stained with ethidium bromide. (Panel b): Southern hybridization with $\left[{ }^{32} \mathrm{P}\right]$ labeled TPM1exon 3 probe. (Panel c): Amplified DNA with TPM1 $\kappa$ specific primer pair [MTPM1-2A(+)/MTPM1$9 \mathrm{~A}(-)]$ were separated by agarose gel electrophoresis and stained subsequently with ethidium bromide. (Panel d): Southern hybridization with $\left[{ }^{32} \mathrm{P}\right]$ labeled TPM1 exon 3 probe. For (A) and (B): (Lane 1): RNA from 3 horse heart; (Lane 2): RNA from horse skeletal muscle; (Lane 3): Primer control.

The nucleotide as well as deduced amino acid sequences of TPM1 $\alpha$ and TPM1 1 is shown in Figure 3 and Figure 4, respectively. It is to be noted that the deduced amino acid sequences of horse TPM $1 \alpha$ is $\sim 100 \%$ similar with human, mouse, and bovine TPM1 $\alpha$ sequences (Table 2), whereas there are four amino acid changes in horse exon 2a (Fig. 5), which is the integral part of TPM $1 \kappa$ isoform. In order to determine the expression of TPM $1 \kappa$ protein in horse heart and skeletal muscle, we used a TPM $1 \kappa$ specific antibody that was raised in rabbits against a 15-mer peptide from the exon 2a of human TPM1к (Rajan et al., 2010) (Fig. 6). Unfortunately, we failed to detect TPM1к expression both in hearts and skeletal muscle of horse. It is to be noted that in horse TPM1 1 , there are three changes in

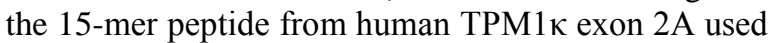
for raising antibody in rabbits (Fig. 6). Hence, antibody

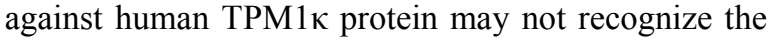

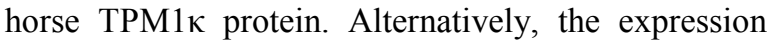
level of TPM1 $\kappa$ protein is extremely low in equine striated muscle used in this study. 


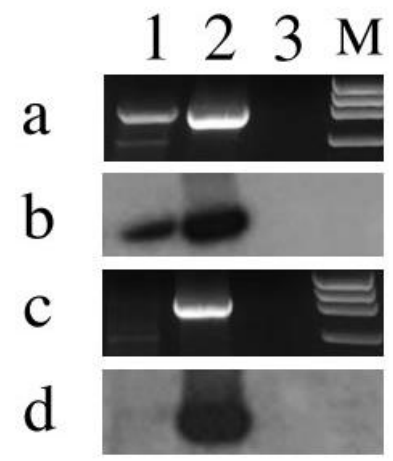

Fig. 3. Agarose gel electrophoresis of amplified TPM $2 \alpha$ and TPM $3 \alpha$ DNAs by conventional RT-PCR. (Panel a): The PCR amplified DNA with TPM2a specific primer-pair MTPM21A(+)/MTPM9A(-) were separated by agarose gel electrophoresis and subsequently stained with ethidium bromide. (Panel b): Southern hybridization with $\left[{ }^{32} \mathrm{P}\right]$ labeled TPM2 exon 3 probe Table 1). (Panel c): The PCR amplified DNA with TPM3a specific primer-pair MTPM31A(+)/MTPM3-9A(-) were separated by agarose gel electrophoresis and subsequently stained with ethidium bromide. (Panel d): Southern hybridization with $\left[{ }^{32} \mathrm{P}\right]$ labeled TPM 3 exon 2 probe (Table 1). (Lane 1): RNA from 3 horse heart; (Lane 2): RNA from horse skeletal muscle; (Lane 3): Primer control.

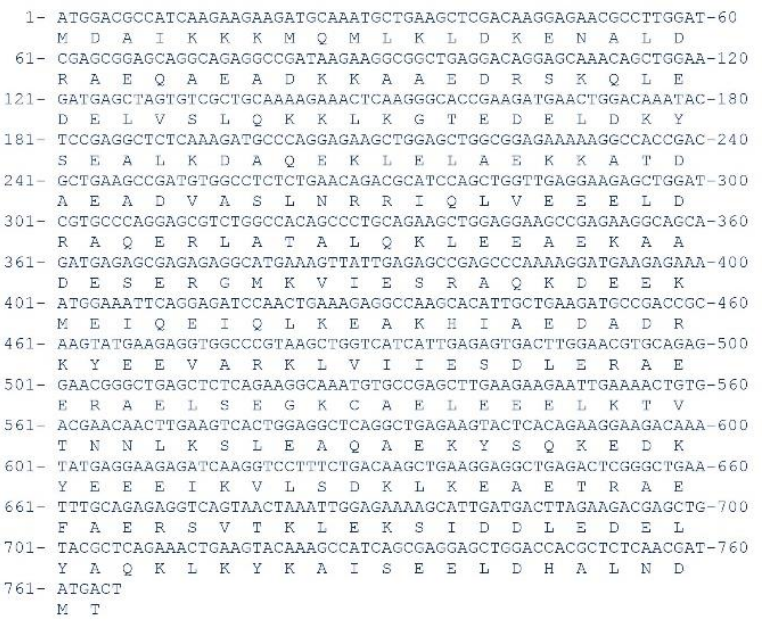

Fig. 4. Nucleotide and deduced amino acid sequences of Horse TPM1 $\alpha$. Nucleotide sequence of the PCR amplified DNA with the primer-pair MTPM1-1A (+)/TPM1-9A (-) as well as the cloned cDNA displayed the same nucleotide sequence as shown above. The deduced protein/amino acid sequences appear below the nucleotide sequence.

\section{Cloning and sequencing of TPM $2 \alpha$ and TPM $3 \alpha$ using conventional RT-PCR}

Primer-pairs used for amplification of TPM $2 \alpha$ and TPM3 $\alpha$ by RT-PCR with RNA from heart and skeletal muscle, designed by aligning various published mammalian and non-mammalian TPM2 and TPM3 sequences, are listed in Table 1.

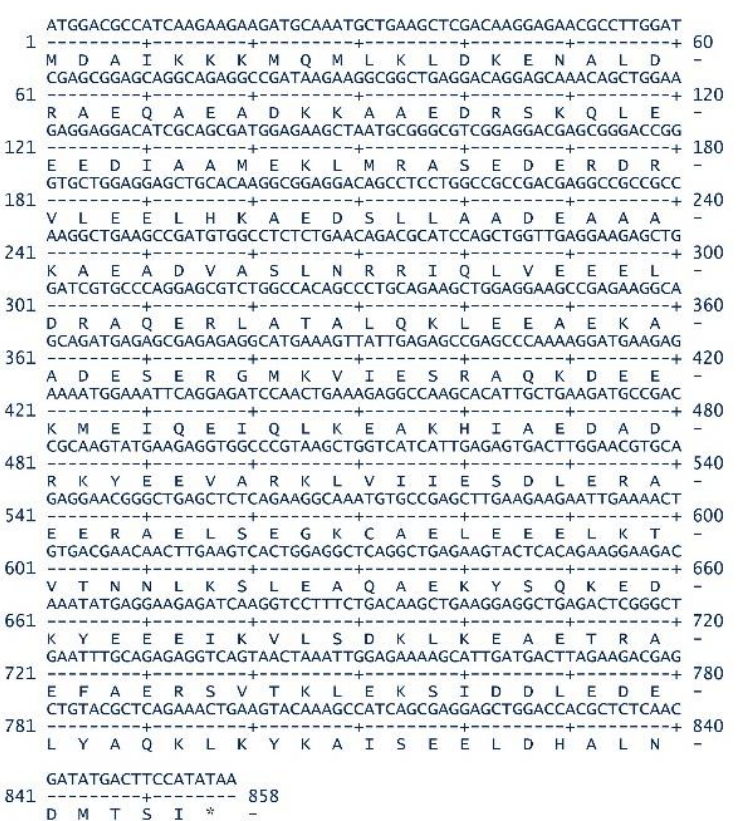

Fig. 5. Nucleotide and deduced amino acid sequences of Horse ТРМ1к. The deduced protein/amino acid sequences appear below the nucleotide sequences. It is to be noted that the copy number of TPM $1 \kappa$ compared to the copy number of TPM1 $\alpha$ is significantly lower. Hence, using the T/A cloning system, it was extremely difficult to get the full length TPM1 $\kappa$ cDNA clone from the amplified products using generic

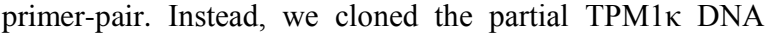
(amplified by P1/P2 primer-pair as shown in Fig. 1) into T/A cloning vector and sequenced it. The rest of the N-terminus sequencing was done as follows: cDNAs were made with RNA from horse heart and skeletal muscle made using P2 primer, which is located in exon 9a/b (Fig. 1) and the amplification was done with the two sets of primer-pairs P1/P7 and P4/P6. The amplified DNAs were separated by agarose gel electrophoresis and stained with ethidium bromide. DNA was extracted from the desired band using gel extraction kit as described under the Methods section. Isolated DNAs were sequenced from both sides with appropriate oligonucleotides.

41

80

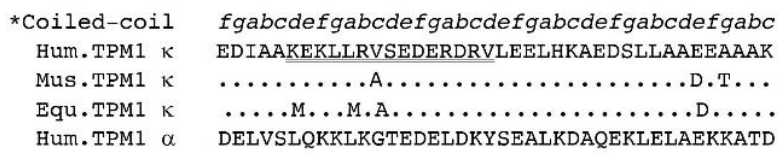

Fig. 6. Alignment of the peptide sequences encoded by exon $2 \mathrm{a}$ of the TPM1 gene from human, horse, and mouse. Double underlined 15 mer peptide of human TPM1 $\kappa$ is the peptide used for raising antibody (Rajan et al., 2010). Please note the differences in the amino acid residues between different species. For comparison, we have included the TPM1.exon $2 b$ sequences (part of TPM1 $\alpha$ ) also at the end. (*) Position refers to the location in leucinezipper coiled-coil motif; $a$ and $d$ are the interface residues; $e$ and $g$ are outer residues that may interact across the coiled-coil; $b, c$ and $f$ are on the far side from the coiled-coil interface (Rajan et al., 2010). 
Table 2. Percent similarity in nucleic acid as well as amino acid sequences of different sarcomeric isoforms from horse, human, bovine, and monkey

\begin{tabular}{|c|c|c|c|}
\hline \multirow{2}{*}{$\begin{array}{l}\text { Gene/ } \\
\text { Isoform }\end{array}$} & \multirow{2}{*}{ Comparison } & \multicolumn{2}{|c|}{$\%$ Similarity } \\
\hline & & N.A. & A.A. \\
\hline \multirow{4}{*}{ TPM1 $\alpha$} & Horse v Human & 95.98 & 99.647 \\
\hline & Horse v Mouse & 93.86 & 100 \\
\hline & Horse v Bovine & 95.63 & 99.647 \\
\hline & Horse v Monkey & 96.15 & 100 \\
\hline \multirow{2}{*}{ ТРМ1к } & Horse v Human & 95.32 & 98.59 \\
\hline & Horse v Mouse & 93.22 & 98.94 \\
\hline \multirow{4}{*}{ TPM $2 \alpha$} & Horse v Human & 95.07 & 99.647 \\
\hline & Horse v Mouse & 94.836 & 100 \\
\hline & Horse v Bovine & 95.775 & 100 \\
\hline & Horse v Monkey & 95.657 & 99.647 \\
\hline \multirow{4}{*}{ ТРМ $3 \alpha$} & Horse v Human & 97.427 & 100 \\
\hline & Horse v Mouse & 92.723 & 98.947 \\
\hline & Horse v Bovine & 97.066 & 100 \\
\hline & Horse v Monkey & 96.959 & 100 \\
\hline
\end{tabular}

(N.A.): Nucleic acid; (A.A.): Amino acids.

The amplified DNAs were run in agarose gel as stated under TPM1 $\alpha$. The bands of appropriate size(s) (not shown) were gel eluted and sequenced. The nucleotide sequence data confirmed the corresponding sarcomeric tropomyosin expressed in heart and skeletal muscle. Expression analyses were carried out by qRT-PCR using relative expression and copy number determination as given in the following section. Amplified DNA was also cloned in a T/A cloning vector and a particular isoform was detected by PCR using an isoform specific primer-pair as described under TPM $1 \alpha$ and TPM1 $\kappa$. DNA for TPM $2 \alpha$ and TPM $3 \alpha$ were isolated from the colony carrying the plasmid with the particular amplified DNA.

Isolated DNAs were subsequently used for determining the nucleotide sequences. The nucleotide as well as deduced amino acid sequences for TPM $2 \alpha$ and TPM $3 \alpha$ are given in Figure 7 and Figure 8, respectively. Comparison of nucleotide as well as deduced amino acid sequences with other mammalian TPM $2 \alpha$ and TPM $3 \alpha$ sequence is presented in Table 2. Similarity in amino acid sequences of horse TPM $2 \alpha$ with human and other mammals is near 100 percent and the percent similarity at the nucleotide level is $\sim 95 \%$. Similarly, the nucleotide as well as deduced amino acid sequences of horse TPM $3 \alpha$ when compared to other mammalian system, are also very conserved. At the amino acid level they are about $100 \%$ similar.

Relative expression of TPM1 $\alpha, T P M 1 \kappa, T P M 2 \alpha$, and TPM3 $\alpha$ in horse heart and skeletal muscle

qRT-PCR analyses were carried out using the 2-(ddCt) method to determine the relative expression of TPM1 $\alpha$ or TPM1 $\kappa$ and $18 \mathrm{~S}$ rRNA was used as the reference gene in making this determination.

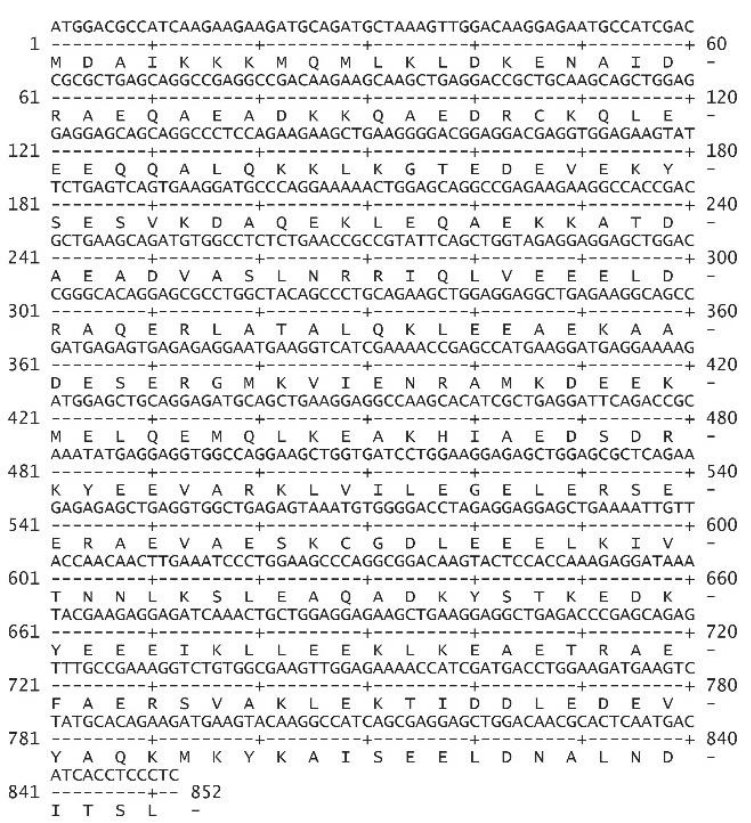

Fig. 7. Nucleotide and deduced amino acid sequences of the Horse TPM $2 \alpha$.

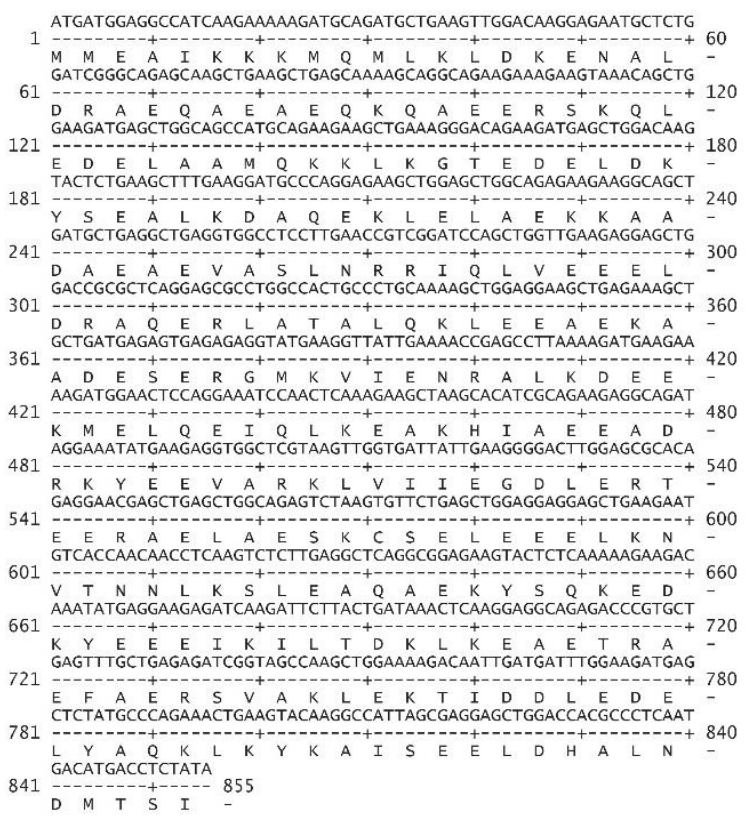

Fig. 8. Nucleotide and deduced amino acid sequences of the Horse ТPM $3 \alpha$.

In order to eliminate the effect of other TPM isoforms, the cDNA was made with a gene and isoform specific primers.

The results show the unique melting temperatures for TPM1 $\alpha$ (top, Fig. 9A) relative to TPM1к (bottom). Agarose gel electrophoresis was also performed with the qRT-PCR amplified DNA of both these isoforms (Fig. 9B). Ethidium bromide staining shows a single band for each amplified DNA. 


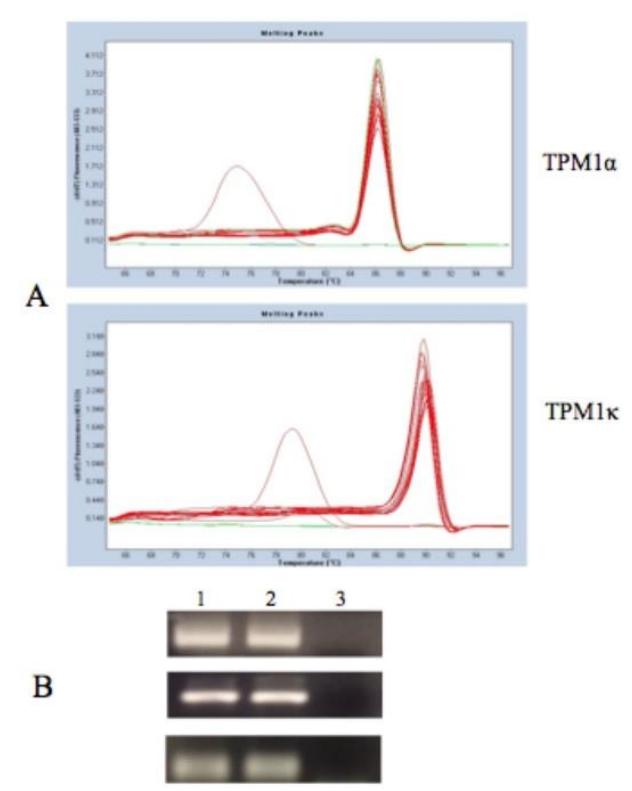

Fig. 9. Melt curves of qRT-PCR and the agarose gel electrophoresis of PCR amplified DNA stained with ethidium bromide. (A): Melt curves for amplified horse TPM1 $\alpha$ and TPM $1 \kappa$ DNA. The melting points of TPM $1 \alpha$ and TPM $1 \kappa$ are different. The multiple curves represent the products from multiple replicates of the RT-PCR assay. (B): PCR products separated by agarose gel electrophoresis and subsequently stained with ethidium bromide. The results demonstrate amplification of a single product for TPM1 $\alpha$ (top panel) or TPM1 (middle panel) or 18S rRNA amplification (bottom panel). (Lane 1): Heart; (Lane 2): Skeletal muscle; (Lane 3): Primer control.

The results indicate that the primer-pairs used for amplification for TPM1 $\alpha$ or TPM1 $\kappa$ are specific. Panel A in Figure 10 depicts that TPM1 $\alpha$ expression in horse skeletal muscle is $\sim 1.65$ fold higher compared to cardiac muscle $(\mathrm{p}=0.02578)$. On the contrary, the expression of TPM1 $\kappa$ is a little higher in heart when compared to skeletal muscle (Fig. 10, Panel B). However, the small difference is statistically significant as determined by Student's T-Test $(\mathrm{p}=0.01859)$.

We also performed qRT-PCR for determining the relative expression level of TPM $2 \alpha$ and TPM3 $\alpha$ transcripts using the $2^{\text {DDCT }}$ method. First we determined the efficiency of each of the primer pairs used for the amplification of TPM $2 \alpha$ and TPM $3 \alpha$ respectively. We then determined the melt curve for each isoform, and also analyzed the size of each amplified DNA by agarose gel electrophoresis followed by ethidium staining (Fig. 11).

Finally, we performed the qRT-PCR amplification for determining relative expression. The results as depicted in Figure 12 show that expression of TPM2 $\alpha$ is $\sim 3.6$ fold higher in horse skeletal muscle compared to cardiac muscle.
A

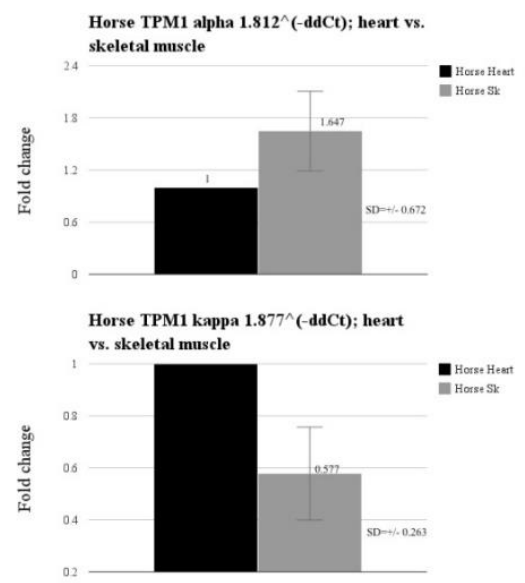

Fig. 10. Relative Expression of TPM $1 \alpha$ and TPM1 $\kappa$ transcripts in heart and skeletal muscle by qRT-PCR. (A): Fold change of TPM1 $\alpha$ in skeletal muscle vs heart Calculated $\mathrm{p}$ value as determined by Student's T-test $=0.025$. (B): Fold change of TPM $1 \kappa$ in skeletal muscle vs heart Calculated $\mathrm{p}$ value as determined by Student's T-test $=0.0189$.

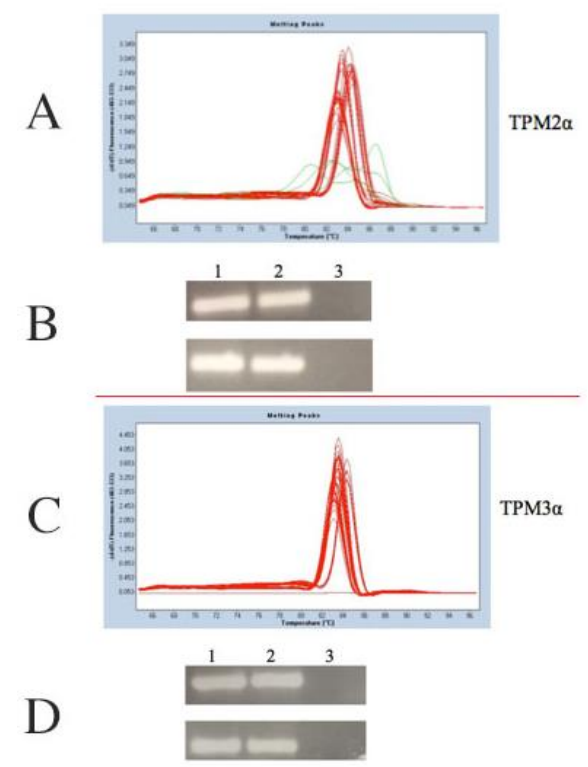

Fig. 11. Melt curves of qRT-PCR and the agarose gel electrophoresis of PCR amplified DNA stained with ethidium bromide. (A): Melt curves for amplified horse TPM $2 \alpha$ DNA. The multiple curves represent the products from multiple replicates of the RT-PCR assay. (B): PCR products separated by agarose gel electrophoresis and subsequently stained with ethidium bromide. The results demonstrate amplification of a single product for TPM $2 \alpha$ (top panel) or $18 \mathrm{~S}$ rRNA amplification (bottom panel). (C): Melt curves for amplified horse TPM $3 \alpha$ DNA. The multiple curves represent the products from multiple replicates of the RT-PCR assay. (D): PCR products separated by agarose gel electrophoresis and subsequently stained with ethidium bromide. The results demonstrate amplification of a single product for TPM $2 \alpha$ (top panel) or 18S rRNA amplification (bottom panel). For (B) and (D): (Lane 1): Heart; (Lane 2): Skeletal muscle; (Lane 3): Primer control. 

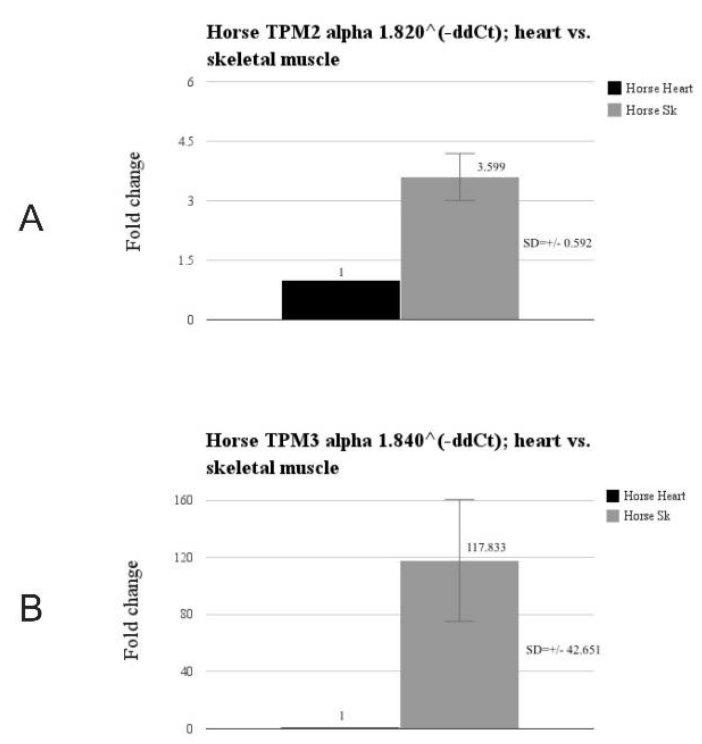

Fig. 12. Fold changes (primer efficiency $(e f f .)^{\wedge}$-ddCt) of horse TPM $2 \alpha$ and TPM $3 \alpha$ in heart $(\mathrm{H})$ vs. skeletal muscle (Sk). (A): Horse TPM $2 \alpha$ eff. $=1.820$ (mean value for 2 separate experiments each being in triplicate). (B): Horse TPM $3 \alpha$ eff. $=1.812$ (mean value for 2 separate experiments each being in triplicate).

However, the relative expression of TPM3 $\alpha$ is about 117 fold higher in skeletal muscle compared to heart. The relative expression experiment using $2^{\text {DDCT }}$ shows that the expression level of most of the sarcomeric TPM isoforms is higher in equine skeletal muscle compared to heart except for the expression of ТРМк, which is higher in cardiac muscle. In order to verify the results we determined the absolute copy number of various TPM isoforms in heart and skeletal muscles. To determine the absolute copy number of a particular isoform we had to use an isoform specific cloned cDNA as the template for calculating the standard curve. We also determined the standard curve for TPM $2 \alpha$ and TPM $3 \alpha$ using respective cDNA template and isoform specific primer-pair (Fig. 11).

Determination of the absolute copy number of transcripts of TPM1 in equine heart and skeletal muscle

The results presented in Table 3 show the absolute copy

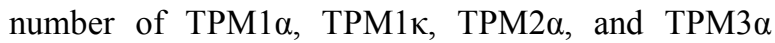
expressed in heart and skeletal muscle. The results are in good agreement with our results on relative expression as presented in the preceding section. In brief, the copy numbers of each of three isoforms, TPM1 $\alpha$, TPM $2 \alpha$, and TPM $3 \alpha$ are higher in skeletal muscle. On the contrary, the copy number of TPM1 $\kappa$ is higher in heart. It is to be noted that the expression level of TPM $1 \kappa$ in equine heart is very low $\left(1.34 \times 10^{2} / \mu \mathrm{g}\right.$ of total RNA) and it is even lower in skeletal muscle.
Western blot analyses for detection of sarcomeric tropomyosin expression in equine heart and skeletal muscle.

Our Western blot results with extracts from horse hearts and skeletal muscle using the $\mathrm{CH}-1$ monoclonal antibody that recognizes all sarcomeric tropomyosin proteins, shows the expression in hearts (lane 1, panel b, Fig. 13).

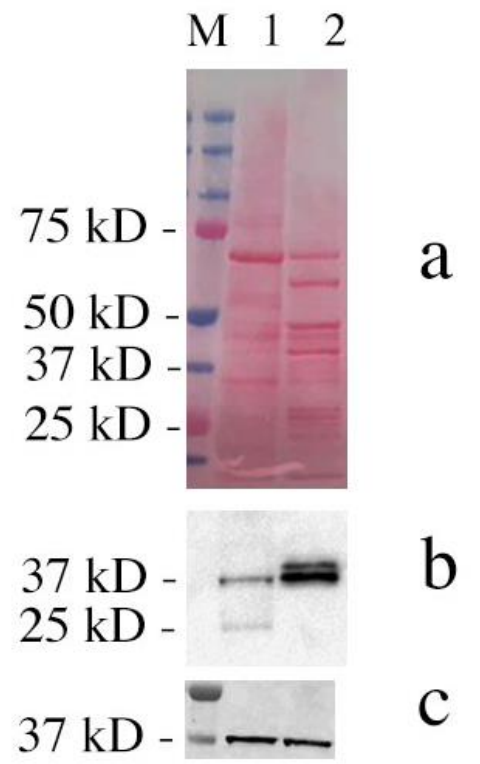

Fig. 13. Western blot analyses of sarcomeric tropomyosin in horse heart and skeletal muscle extracts. (Panel a): Ponceu staining of the blot. (Panel b): Staining with $\mathrm{CH} 1$ monoclonall antibody. (Panel c): staining with anti-GAPDH antibody. (Lane M): Molecular weight marker; (Lane 1): Heart ; (Lane 2): Skeletal muscle.

Because $\mathrm{CH} 1$ recognizes all sarcomeric isoforms for

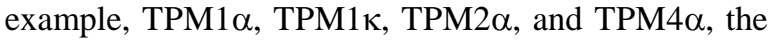
results do not help determine expression of a particular TPM isoform. As mammalian hearts are known to generate overwhelming amount of TPM1a ( 95\% of the total sarcomeric TPMs) (Rajan et al., 2010), one can assume that the $\sim 37 \mathrm{kD}$ band represents TPM1 $\alpha$. At this point it is extremely difficult to assign an identity to the ${ }^{`} 25 \mathrm{kD}$ band.

To the best of our knowledge, sarcomeric tropomyosin is not low molecular weight TPM. However, there are reports in the literature and also our unpublished results that strongly support that the low molecular weight TPM with exon 9a are expressed in mammalian striated muscles. As a matter fact, the predicted sequence of tropomyosin alpha-1 chain isoform X1 with exon 9a of horse is available in the data base (NCBI Reference Sequence: XP_005603060.1). It is possible that the low molecular band may represent such an isoform. 


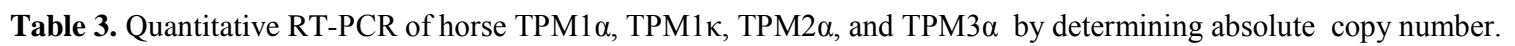

\begin{tabular}{|c|c|c|c|c|c|c|}
\hline Tissue / organ & TPM1 $\alpha$ & ТРМ1к & TPM $2 \alpha$ & TPM3к & $\begin{array}{c}\text { Ratio } \\
\text { TPM1 } \alpha: \text { TPM } 2 \alpha\end{array}$ & $\begin{array}{c}\text { Ratio } \\
\text { TPM1 } \alpha: \text { TPM } 3 \alpha\end{array}$ \\
\hline Heart & $\begin{array}{c}4.25 \times 10^{\wedge} 4+ \\
9.1 \times 10^{\wedge} 2\end{array}$ & $1.34 \mathrm{X} 10^{\wedge} 2$ & $\begin{array}{c}2.43 \times 10^{\wedge} 5+ \\
2.2 \times 10^{\wedge} 3\end{array}$ & $\begin{array}{l}1.93 \times 10^{\wedge} 3 \\
+1.1 \times 10^{\wedge} 2\end{array}$ & 0.17 & 22 \\
\hline Sk muscle & $\begin{array}{c}1.86 \times 10^{\wedge} 4+ \\
8.4 \times 10^{\wedge} 2\end{array}$ & $0.46 \times 10^{\wedge} 2$ & $\begin{array}{c}2.47 \times 10^{\wedge} 6+ \\
1.9 \times 10^{\wedge} 4\end{array}$ & $\begin{array}{c}1.54 \times 10^{\wedge} 6 \\
2.1 \times 10^{\wedge} 4\end{array}$ & 0.007 & 0.012 \\
\hline $\begin{array}{l}\text { Ratio } \\
\mathrm{H}: \mathrm{Sk}\end{array}$ & 2.28 & 2.9 & 0.098 & 0.001 & & \\
\hline
\end{tabular}

Sarcomeric tropomyosin expression in equine skeletal muscle (lane 2, panel b, Fig. 13) is relatively higher compared to heart. Multiple bands are also visible in the skeletal muscle - the higher band is $\sim 39 \mathrm{kD}$ and the lower band $\sim 37 \mathrm{kD}$. The broadness of the lower band $(\sim 37 \mathrm{kD})$ suggests the presence of more than one protein/iosform. It is to be noted that the higher band in

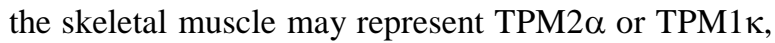
each of which run slower compared to TPM $1 \alpha$ or TPM $3 \alpha$. The lower band may represent TPM $1 \alpha$ and TPM $3 \alpha$ because the electrophoretic mobility of the two are similar (Pieples and Wieczorek, 2000; Rajan et al., 2010). The results with CH1 monoclonal antibody show that the overall sarcomeric TPM protein expression in equine skeletal muscle is higher compared to heart. while GAPDH expression is similar in heart and skeletal muscle. (compare lane 1 and 2, panel c, Fig. 13).

\section{Discussion}

Tropomyosin (TPM) belongs to a multigene family of actin binding proteins. In vertebrates, tropomyosins are encoded by four genes designated as TPM1, TPM2, $T P M 3$, and TPM4, which generate more than 50 isoforms via alternate splicing and/or using different promoters. To the best of our knowledge no sarcomeric tropomyosin has been cloned and sequenced from an equine species. Nor has the full isoform expression repertoires been defined. Hence, we undertook a comprehensive study on the characterization of all sarcomeric tropomyosins from horse hearts and skeletal muscle.

Sarcomeric tropomyosin includes TPM1 $\alpha$ and TPM1 1 , which are the alternatively spliced products of the TPM1 gene. The difference between these two isoforms is in exon 2. TPM1 $\alpha$ contains exons $1 \mathrm{a}, 2 \mathrm{~b}, 3,4,5,6 \mathrm{~b}$, $7,8,9 \mathrm{a} / \mathrm{b}$ whereas TPM1 $\kappa$ includes all the exons as in TPM1 $\alpha$ except for exon $2 b$; instead it has exon $2 a$. The sarcomeric isoform of TPM 2 and TPM 3 are designated as TPM $2 \alpha$ and TPM $3 \alpha$, respectively.

In this study, we have not included TPM4 because the role(s) of TPM $4 \alpha$ in muscle contraction is not well defined in mammals as in the case of avian (Fleenor $e t$ al., 1992), amphibians (Hardy et al., 1995; Spinner et al., 2002), and aquatic animals like fish (Schevzov et al., 2011). In some mammals for example in rodents, TPM4 $\alpha$ is not expressed. In humans, however, we have recently reported the expression of TPM $4 \alpha$ transcripts in hearts and skeletal muscle. In fact, we have also reported the potential of TPM $4 \alpha$ protein expression in human hearts (Spinner et al., 2002). However, we do not know yet the function it may perform in cardiac contractility of human striated muscles.

For the first time, we have cloned and sequenced TPM1 $\alpha$ and ТРM1 $\kappa$ from horse heart and skeletal muscle. Interestingly, the amino acid sequences of horse TPM $1 \alpha$ is $\sim 100 \%$ similar with human TPM1 $\alpha$ isoform (Fig. 6). As there are some differences in exon 2a sequences of horse and human TPM1 gene, there are some differences in ТРM1 $\kappa$ from human and horse. Unfortunately, the nucleotide as well as amino acid sequences of TPM1 $\alpha$ from Equus caballus (the horse species that includes all domestic horses) has not been reported in GenBank. Recently predicted sequences of two TPM1 isoforms from $\mathrm{E}$ caballus have been posted in the GenBank; neither of them can encode a high molecular wt tropomyosin like TPM1 $\alpha$.

However, the amino acid sequences of the Predicted TPM1isoform X 1 (XP_008520892.1; submitted in July 2014) from Equus przewalskii (another horse species that still lives in the wild of Central Asia) shows $100 \%$ similarity with our TPM1 $\alpha$ sequence from Equus caballus. Seven predicted TPM1 isoform sequences from Equus przewalskii (known as Przewalski horse) are available in GenBank. Unfortunately none of the seven isoforms have exon 2a like sequences. So, we cannot compare our TPM1к sequence from Equus caballus with predicted TPM1 $\kappa$ from Przewalski horse. Tropomyosin is an alpha helical coiled-coil dimeric protein. The coiled-coil structure is characterized by a heptapeptide repeat motif (a-b-c-d-e-f-g). The amino acid residues in the hydrophobic core positions a and $d$ are the primary determinants of folding and stability as explained by Rajan et al. (2010).

The difference between TPM $1 \alpha$ and TPM $1 \kappa$ is only in exon2. TPM1 $\alpha$ contains exon $2 b$ where as TPM1 $\kappa$ contains exon 2a. Hence, 26 out of 40 amino acid residues are different between human TPM $1 \alpha$ and ТРМ1к. As pointed out earlier, 284 amino acid 
residues are identical in human and horse TPM1 $\alpha$. Although the TPM1 $\alpha$ from horse and human are identical and exon 2a in horse contain four additional substitutions, the stability of the coiled-coil structure for TPM1 $\alpha$-TPM1 $\kappa$ hetero dimer as well as TPM1 $\kappa$ ТРM1 $\kappa$ homodimer may be altered. For example, L43I substitution in human TPM1 $\kappa$ in position $a$ of the heptad may slightly destabilize of the $\kappa / \kappa$ coiled coil relative to the $\alpha / \alpha$ coiled coil. Also, this substitution causes the loss of hydrophobicity.

In horse, the same substitution will have the similar effect forming $\alpha-\kappa$ coiled coil heterodimer. L46M in position $d$ unlike in humans, there will be lesser loss of the hydrophobicity and the destabilization will also be less. No SB (salt bridge) will be added like in the case of human. In the case of L50M in position " $a$ " may cause some loss of hydrophobicity. V52A in a position of $c$ may not affect significantly the coiled coil structure in horse TPM1к. E75D in position $e$ may not change the coiled coil structure. K77T in position $g$ may stabilize the structure may still lose the hydrophobicity. At this point it's worth mentioning that the different 26 out of 40 amino acids in exon 2 changes the biding affinity of TPM1 $\kappa$ with F-actin.

In the absence of troponins, the binding is significantly lower compared to the binding of F-actin with TPM $1 \alpha$. In fact, no biding between TPM $1 \kappa$ and F-actin was observed in the absence of troponins. However, no differences were observed for the binding of TPM1 $\alpha$ or TPM1 $\kappa$ with F-actin in the presence of troponins in vitro (Rajan et al., 2010). We are planning to perform similar experiments with bacterially expressed TPM1 $\alpha$ and ТРМ1 $\kappa$ with $\mathrm{F}$-actin in vitro in future.

At this juncture one of our observations deserves added attention. The 41-mer peptide encoded by exon $2 \mathrm{~b}$, which is the integral part of TPM1 $\alpha$ is highly conserved not only among mammals but most likely in all vertebrates from fish to humans (Fig. 6). On the contrary, the 4-mer peptide sequences encoded by exon $2 \mathrm{a}$, which is a component of TPM1 $\kappa$, have not been conserved throughout vertebrate evolution (Fig. 6). Twenty five years ago David Helfman had the same observation although at that time exon $2 \mathrm{a}$ was known to be part of TPM $1 \beta$ and exon $2 b$ as part of TPM $1 \alpha$ (Lees-Miller and Helfman, 1991). Currently, exon 2a is also known to be an integral part of the sarcomeric isoform ТРM1 $\kappa$. Although functional involvement of the tropomyosin isoforms containing the exon $2 \mathrm{a}$ peptide has been broadened, actin is still the only protein that is known to bind to this region of tropomyosin.

The fundamental implication and the reasons for the large divergence in amino acid residues 39-80 of TPM $1 \kappa$ and /or TPM $1 \beta$ remains a mystery as stated by
David Helfman (Lees-Miller and Helfman, 1991). TPM $2 \alpha$ protein sequences from Equus caballus are identical with the predicted TPM2 isoform X 1 published in GenBank (XP_003364171). Our TPM2 $\alpha$ protein sequence from horse is almost identical with human and other mammalian TPM2 $\alpha$ (Table 2). Finally, our TPM $3 \alpha$ protein sequence is identical with Predicted tropomyosin alpha-3 chain isoform X1 [Equus caballus] (XP_005610140 submitted in November 2015) and also 97-98\% similar to human and other mammalian TPM $3 \alpha$ sequences (Table 2).

We performed expression analyses of the transcripts of each of these isoforms with RNA from heart and skeletal muscle by qRT-PCR using two different approaches - relative expression and by determining absolute copy number using a standard curve of the corresponding cloned cDNA as a template. The results from both assays are comparable. Our results indicate that the relative expression of TPM1 $\alpha$ is higher in skeletal muscle compared to heart.

On the other hand the relative expression of TPM1 $\kappa$ is higher in the heart. The results are consistent with our reported findings in mice (Dube et al., 2014), and axolotl (Thomas et al., 2010). However, when we compared the absolute copy number expression in heart and skeletal muscle, the expression of TPM1 $\alpha$ compared to TPM1 $\kappa$ is much higher ( 300 fold) in heart. In skeletal muscle, the expression of TPM1 $\alpha$ compared to TPM1 $\kappa$ is $\sim 4000$ fold higher. In other words the copy number of TPM1 $\kappa$ both in heart and skeletal muscle is very low compared to TPM1 $\alpha$. However, the expression of TPM $1 \kappa$ is $\sim 2.9$ fold higher in heart whereas the expression of TPM1 $\alpha$ in skeletal muscle compared to heart is $\sim 4.5$ fold higher. This is consistent with published results in other systems (Booth et al., 1998).

Interestingly, the expression level of transcripts of TPM1 $\alpha$ and TPM1 $\kappa$ is comparable in human hearts unlike in Equine system. However, at the protein level, TPM1 $\alpha$ constitutes $\sim 90 \%$ of the total sarcomeric trpomyosin whereas TPM1 $\kappa$ is $\sim 5 \%$. There is a sense that a control mechanism is operative at the translation level that determines the efficiency and/or priority of the various TPM transcripts (Denz et al., 2004; Dube et al., 2016). It's worth mentioning at this point that Rajan et al. (2010) generated transgenic mice overexpressing ТРМ1 $\kappa$ in a cardiac-specific manner that led to a concomitant decrease in TPM1 $\alpha$ protein without affecting the level of total sarcomericTPM1 proteins. Incorporation of increased levels of TPM1 1 protein in myofilaments leads to dilated cardiomyopathy (DCM). Physiological alterations include decreased fractional shortening, systolic and diastolic dysfunction, and decreased myofilament calcium sensitivity with no 
change in maximum developed tension. Also, they found that the level of expression of TPM1 $\kappa$ is increased in human dilated cardiomyopathy and heart failure.

In order to illustrate biological significance for expressing the TPM1k isoform these authors suggested that in terminally differentiated cells such as cardiomyocytes, there is a need for adaptation to changing environments. Increasing protein isoform diversity through processes such as alternative splicing meets this need. The TPM $1 \kappa$ isoform, one of the several products of the TPMI gene (Dube et al., 2014), might provide the opportunity to modulate sarcomeric performance during changing conditions such as exercise, stress, or cardiac disease.

We now speculate that it is most likely beneficial for the athletic activity of Equines to having a lower level of TPM $1 \kappa$ in heart. However, we must admit that we do not know whether TPM1 $\kappa$ protein is expressed in equine striated muscle. Also, it is worth trying to create

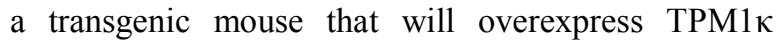
protein in skeletal muscle. This may also give some

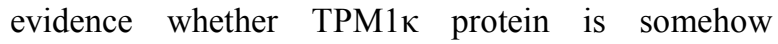
associated with animal athletic activity. However, the functional role of TPM1 $\kappa$ is yet to be established. It is

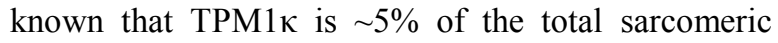
tropomyosin protein in human hearts, which is also true for axolotl (Thomas et al., 2010).

We already reported that an anti-sense mediated down regulation of TPM1 $\kappa$ inhibited the cardiac contractility in axolotl in situ suggesting a critical role of TPM1 $\kappa$ protein in cardiac contractility. However, we do not know whether this is true for other vertebrates like humans. The expression of TPM $2 \alpha$ and TPM $3 \alpha$ in horse skeletal muscle is higher compared to cardiac muscle. Our findings are consistent with the published information in other mammalian systems. It is long been known that TPM $3 \alpha$ transcripts are expressed in hearts and skeletal muscle.

It was not known that TPM $3 \alpha$ protein is expressed in human hearts. Recently, Marston et al. (2013) showed that TPM $3 \alpha$ protein is expressed in human hearts in lower quantity $(<5 \%$ of the total sarcomeric TPM protein).

Our unpublished data also support this observation. TPM $3 \alpha$ transcripts have not been detected in mouse hearts. Although TPM $3 \alpha$ transcripts are expressed in equine hearts and skeletal muscle, we do not know the expression patterns of the corresponding protein.

\section{Acknowledgements}

The work was supported by a grant from the Department of Medicine and Barbara Kopp Cancer Research Fund to BJP and funding from College of Health Professionals, Upstate Medical University,
Syracuse, to DKD. Authors are thankful to Ms. Lori Spicer for her assistance in the preparation of the manuscript.

\section{Conflict of interest}

The authors declare that there is no conflict of interests.

\section{References}

Booth, F.W., Tseng, B.S., Fluck, M. and Carson, J.A. 1998. Molecular and cellular adaptation of muscle in response to physical training. Acta Physiol. Scand. 162(3), 343-350.

Denz, C.R., Narshi, A., Zajdel, R.W. and Dube, D.K. 2004. Expression of a novel cardiac-specific tropomyosin isoform in humans. Bioch. Biophys. Res. Commun. 320(4), 1291-1297.

Dube, D.K., Dube, S., Abbott, L., Alshiekh-Nasany, R., Mitschow, C. and Poiesz, B. 2016. Cloning, sequencing, and the expression of the elusive sarcomeric TPM4 $\alpha$ isoform in humans. Mol. Biol. Int. doi:10.1155/2016/3105478.

Dube, S., Panebiancol, L., Matoq, A.A., Chionuma, H.N., Denz, C.R., Poiesz, B.J. and Dube, D.K. 2014. Expression of TPM1k, a novel sarcomeric isoform of the TPM1 gene in mouse heart and skeletal muscle. Mol. Biol. Int. 2014:896068.doi: 10.1155/2014 /896068.Epub.

Eivers, S.S., McGivney, B.A., Fonseca, R.G., MacHugh, D., Menson, K., Park, S.D., Rivero, J.L., Taylor, C.T., Katz, L.M. and Hill, E.W. 2010. Alterations in oxidative gene expression in equine skeletal muscle following exercise and training. Physiol. Genomics 40, 83-93.

Eivers, S.S., McGivney, B.A., Gu, J., MacHugh, D.E., Katz, L.M. and Hill, E.W. 2012. PGC-1 $\alpha$ encoded by the PPARGC1A gene regulates oxidative energy metabolism in equine skeletal muscle during exercise. Anim. Genet. 43, 153-162.

Fleenor, D.E., Hickman, K.H., Lindquester, G.J. and Devlin, R.B. 1992. Avian cardiac tropomyosin gene produces tissue-specific isoforms through alternative RNA splicing. J. Muscle Res. Cell Motil. 13, 55-63.

Gunning, P., O’Neill, G. and Hardmen, E. 2008. Tropomyosin-based regulation of actin cytoskeleton in time and space. Physiol. Rev. 88, 135.

Hardy, S., Theze, N., Lepetit, D., Allo, M.R. and Thiebaud, P. 1995. The Xenopus laevis TM-4 gene encodes non-muscle and cardiac tropomyosin isoforms through alternative splicing. Gene 156, 265-270.

Lees-Miller, J. and Helfman, D. 1991. The molecular basis for tropomyosin isoform diversity. Bioessays 13, 429-437. 
Livak, K.J. and Schmittgen, T.D. 2001. Analysis of relative gene expression data using real-time quantitative PCR and 2-DDCT method. Methods 25, 402-408.

Marston, S.B., Copeland, O., Messer, A.E., MacNamara, E., Nowk, K., Zampronio, C.G. and Ward, D.G. 2013. Tropomyosin isoform expression and phosphorylation in the human heart in health and disease. J. Muscle Res. Cell Motil. 34(3-4), 189-197.

McGivney, B.A., Eivers, S.S., MacHugh, D.E., MacLeod, J.N., O'Gorman, G.M., Park, S.D., Katz, L.M. and Hill, E.W. 2009. Transcriptional adaptations following exercise in thoroughbred horse skeletal muscle highlights molecular mechanisms that lead to muscle hypertrophy. BMC Genomics 10, 638. DOI: 10.1186/1471-2164-10638.

McGivney, B.A., McGettigan, P.A., Browne, J.A., Evans, A.C., Fonseca, R.G., Loftus, B.J., Lohan, A., MacHugh, D.E., Murphy, B.A., Katz, L.M. and Hill, E.W. 2010. Characterization of the equine skeletal muscle transcriptome identifies novel functional responses to exercise training. BMC Genomics 11, 398. DOI: 10.1186/1471-2164-11398.

Nan, C., Dube, S., Matoq, A., Mikesell, L., Abbott, L., Alshiekh-Nasany, R., Chionuma, H., Huang, X., Poiesz, B.J. and Dube, D.K. 2015. Expression of sarcomeric tropomyosin in striated muscles in axolotl treated with shz-1, a small cardiogenic molecule. Cardiovasc. Toxicol. 15(1), 29-40.

Perry, S.V. 2001. Vertebrate tropomyosin, properties and function. J. Muscle Res. Cell Motil. 22, 5-49.

Pfaffl, M.W. 2001. A new mathematical model for relative quantification in realtime RT-PCR. Nucl. Acids Res. 29, 2002-2007.

Pieples, K. and Wieczorek, D.F. 2000. Tropomyosin 3 increases striated muscle isoform diversity. Biochemistry 39, 8291-8297.

Pittenger, M.F., Kazzaz, J.A. and Helfman, D.M. 1994. Functional properties of nonmuscle tropomyosin isoforms. Curr. Opin. Cell Biol. 6, 96-104.

Rajan, S., Jagatheesan, G., Karam, C.N., Alves, M.L., Bodi, I., Schwartz, A., Buclago, C.F., D’Souza, K.M., Akhter, S.A., Boivin, G.P., Dube, D.K., Petrasheveskaya, N., Herr, A.B., Hullin, R., Liggett, S.B., Wolska, B.M., Solaro, R.J. and Wieczorek, D.F. 2010. Molecular and Functional Characterization of a Novel Cardiac-Specific Human Tropomyosin Isoform. Circulation 121, 410-418.

Schevzov, G., Whittaker, S., Fath, T., Lin, J.J. and Gunning, P.W. 2011. Tropomysin isoforms and reagents. Bioarchitecture 1, 135-164.

Spinner, B.J., Zajdel, R.W., McLean, M.D., Denz, C.R., Dube, S., Mehta, S., Choudhury, A., Nakatsugawa, M., Dobbins, N., Lemanski, L.F. and Dube, D.K. 2002. Characterization of a TM-4 type tropomyosin that is essential for myofibrillogenesis and contractile activity in embryonic hearts of the Mexican axolotl. J. Cell Biochem. 85, 747-761.

Thomas, A., Rajan, S., Thurston, H.L., Masineni, S.N., Dube, P., Bose, A., Muthu, V., Dube, S., Wieczorek, D.F., Poiesz, B.J. and Dube, D.K. 2010. Expression of a novel tropomyosin isoform in axolotl heart and skeletal muscle. J. Cell Biochem. $110,875-881$.

Thurston, H.L., Prayaga, S., Thomas, A., Guharoy, V., Dube, S., Poiesz, B.J. and Dube, D.K. 2009. Expression of Nkx2.5 in wild type, cardiac mutant, and thyroxine-induced metamorphosed hearts of the Mexican axolotl. Cardiovas.Toxicol. 9, 13-20.

Yuan, J.S., Reed, A., Chen, F. and Stewart, C.N. 2006. Statistical analysis of real-time PCR data. BMC Bioinformatics 7, 85-101. 